\title{
Wrinkling of a stiff film resting on a fiber-filled soft substrate and its application as tunable metamaterials
}

\author{
Yang Zheng, Guo-Yang Li, Yanping Cao*, Xi-Qiao Feng \\ Institute of Biomechanics and Medical Engineering, AML, Department of \\ Engineering Mechanics, Tsinghua University, Beijing 100084, PR China
}

${ }^{*}$ Corresponding author: Yanping Cao.

Email address: caoyanping@tsinghua.edu.cn; Tel: 86-10-62772520; Fax: 86-10-62781284.

1 


\begin{abstract}
Mechanical self-assembly of ordered patterns via spontaneous buckling of thin-film/soft-substrate systems has received considerable interests in recent years. Here we study the wrinkling of a stiff film resting on a fiber-filled soft substrate. In particular, the effects of the cross-section dimension, spacing, and positions of fibers on the wrinkling patterns in the film/substrate bilayer system are investigated. We show that diverse wrinkling patterns, including sinusoidal wrinkling, period-doubling, period-tripling and mountain ridge modes, may occur at a small or moderate overall compression strain due to the inhomogeneous deformation in the substrate and they can be well controlled by tuning geometrical and physical parameters of the system. To illustrate the potential use of the wrinkling patterns revealed in this study, we investigate the elastic wave propagation in such a wrinkled bilayer using the Bloch wave theory. Our computational results show that diverse stress patterns generated in the soft composites give rise to a rich variety of band structures. Desired bandgaps of elastic waves can be achieved and tuned by simply designing the geometric parameters and controlling the external stimuli imposed to the soft metamaterials.
\end{abstract}

Keywords: Wrinkling; stiff-film/fiber-filled substrate bilayer; stress patterns; soft metamaterial; elastic wave 


\section{Introduction}

Surface wrinkling of a bilayer system consisting of a stiff film and a compliant substrate is ubiquitous in both nature and engineering [1-5]. When a stiff film resting on a homogeneous substrate is subjected to uniaxial compression, sinusoidal surface wrinkling may occur to minimize the total potential energy of the system (Fig. 1a). Further compression may lead to the transition of the wrinkling mode from sinusoidal to period-doubling (Fig. 1b) and the formation of folds (Fig. 1c) [6-9]. When the substrate is subjected to a large pre-stretch before attaching the film, the mountain ridge mode may be observed, as shown in Fig. 1d [10,11]. Wang and Zhao [12] recently reported phase diagrams to predict the different instability modes. Considering the wide applications of the characteristics of wrinkling patterns in film/substrate bilayers, besides the homogeneous substrate, wrinkling of a stiff film on an elastic graded substrate, a soft substrate with interfacial structures and the layered substrates have been addressed [13-17].

Inspired by a recent study of Guttag and Boyce [18] who studied the surface deformation of a soft material enhanced by particles, this paper investigates the wrinkling of a stiff film resting on a fiber-filled soft substrate. We show that diverse wrinkling patterns e.g., sinusoidal strips, period-doubling and period-tripling wrinkles, folds and mountain ridges may occur due to the nonlinear and inhomogeneous deformation in the substrate and they can be well controlled by designing the geometrical parameters of the system, e.g., the cross-section dimension, spacing and 
positions of fibers and/or tuning the modular ratios of the fibers to the soft matrix.

The diverse wrinkling patterns presented here may find broad applications and this letter demonstrates a potential use of the buckled composite system in the design of tunable soft metamaterials. Soft metamaterials may be used as elastic wave guides, vibration controller, acoustic cloaking and ultrasound transducers and have been investigated by many authors during the past decade [19-25]. A unique and interesting feature of soft metamaterials in comparison with hard metamaterials is that the former may undergo large deformation under various external or internal stimuli, and therefore the band structures including the bandgaps can be actively tuned [19-23,26-28].

Alireza and Faramarz [21] and the authors [23,29] have reported that a wrinkled bilayer system may serve a soft metamaterial to control the elastic wave propagation. In this letter, we investigate the propagation of elastic wave in the wrinkled stiff-film/composite-substrate bilayer system. Our finite element simulations show that diverse wrinkling patterns generated in such a composite system not only alter the microstructures, i.e., the geometrical patterns, but also generate diverse periodic stress patterns. Our analysis based on the Bloch wave theory reveals intriguing band structures which largely depend on the material and geometrical parameters (e.g., modular ratios, fiber cross-section dimension, spacing, and positions) and the magnitude of the external load. 


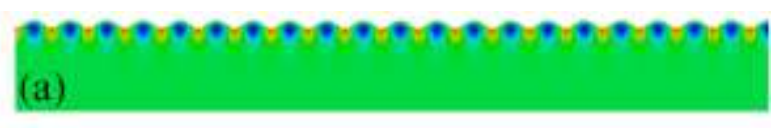

$$
\lambda_{0}=1, \varepsilon_{f}=0.15
$$

(b)

$$
\lambda_{0}=1, \varepsilon_{f}=0.22
$$

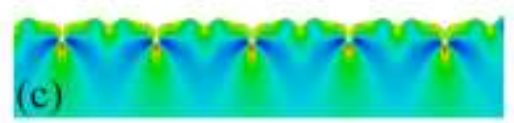

$$
\lambda_{0}=1, \quad \varepsilon_{f}=0.28
$$

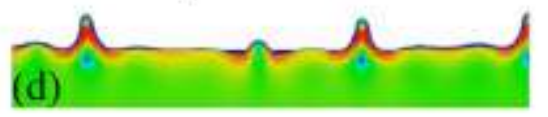

$$
\lambda_{0}=2, \varepsilon_{f}=0.17
$$

Fig. 1 Wrinkling patterns observed in the compression of a stiff film resting on a homogeneous substrate. (a) Sinusoidal wrinkling; (b) period-doubling mode; (c) period-quadrupling and folding; (d) mountain ridge mode.

\section{Model}

The composite system investigated in this study is a stiff film resting on a fiber-filled soft substrate (Fig. 2a), subjected to plane strain compression along $x$ direction. Guttag and Boyce [18] showed that such a soft composite substrate can be obtained with the 3D printing technique. Here both the fibers and the matrix are described with the incompressible neo-Hooke model, with the initial shear moduli $\mu_{\mathrm{p}}$ and $\mu_{\mathrm{m}}$, respectively. The mass densities are $\rho_{\mathrm{p}}$ and $\rho_{\mathrm{m}}$ and the geometrical parameters used to describe the fiber cross-section dimension, spacing and positions relative to the interface are shown in Fig. 2. $t, \rho_{\mathrm{f}}$ and $\mu_{\mathrm{f}}$ are the thickness, mass density and initial shear modulus of the film, respectively. When the nominal compression strain $\varepsilon$ is imposed on the system, surface wrinkling may occur, which 
may not only alter the microstructures but also lead to periodic stress patterns.

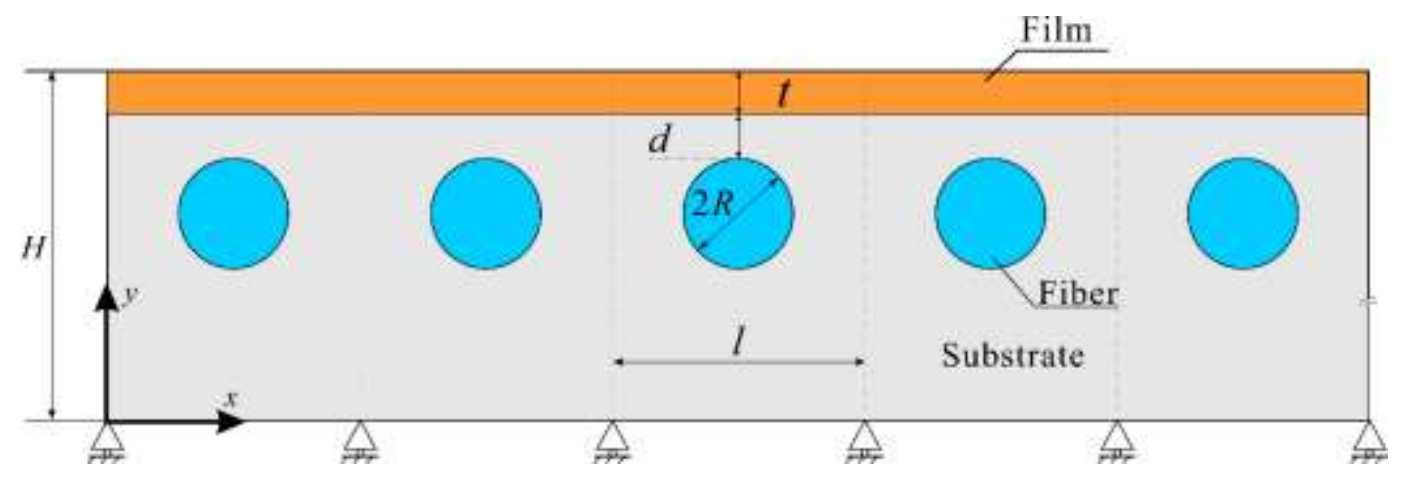

Fig. 2 Mechanical model. $t$ and $H$ are the thicknesses of the film and the bilayer, respectively. $d$ is the distance of the fiber from the interface, $R$ is the radius of the cross-section of fibers, and $l$ determines the spacing of fibers.

\section{Wrinkling patterns in the stiff-film/fiber-filled soft substrate system}

When the bilayer illustrated in Fig. 2 is subjected to uniaxial compression and the compressive strain is greater than a critical value, surface wrinkling will occur. For a homogeneous substrate which is much softer than the film, e.g., $\mu_{\mathrm{f}} / \mu_{\mathrm{m}}=500$ we used in the present study, the critical wrinkling wavelength is determined by [30]

$$
\lambda_{\mathrm{c}}=2 \pi t\left(\mu_{\mathrm{f}} / 3 \mu_{\mathrm{m}}\right)^{\frac{1}{3}}
$$

We develop a finite element model to investigate how the cross-section dimension, spacing and position of fibers affect the surface wrinkling patterns. In our simulations, both stiff fibers and soft fibers are investigated. Plane strain model is used to describe the bilayer subjected to uniaxial compression. Approximately $100000 \mathrm{CPE} 8 \mathrm{RH}$ elements in ABAQUS [31] were used. Displacement-controlled loading procedure was adopted. Critical buckling analysis was first carried out on the bilayer system with the fibers having the same initial shear modulus as the matrix. The critical buckling mode 
scaled by a very small factor $(0.01)$ was introduced into the model as the geometrical imperfections to trigger the post-buckling. For the model considered in this study, i.e., the elastic modulus of the fiber is much larger or smaller than that of the matrix, our post-buckling analysis shows that when the position of fibers is close to the interface, they have marked effects on the surface wrinkling pattern and its evolution. The results in Fig. 3 shows that not only Eq. (1) but also the spacing of fibers determine the wrinkling wavelength. For example, Fig. 3 reveals that when $\bar{l}=l / \lambda_{\mathrm{c}}>1$, the wrinkling wavelength $\lambda_{\mathrm{c}}=l /\lfloor\bar{l}\rfloor$ for both stiff fibers and soft fibers, where \lfloor\rfloor is the floor function. When a stiff film resting on a homogeneous substrate is subjected to a uniaxial compressive strain greater than approximately $18.5 \%$, sinusoidal wrinkling mode will give way to the period-doubling mode [10,32]. Figs. $3 \mathrm{~b}$ and $3 \mathrm{e}$ show that in the composite system, period-doubling modes can occur at much smaller compressive strains. Interestingly, the period-doubling mode revealed in our system as shown in Fig. 3e cannot occur in the case of a homogeneous substrate because it is not energetic favorable in comparison with the period-doubling mode given by Fig. $3 b$. The wrinkling mode in Fig. 3b will evolve into folds upon further compression, whereas that in Fig. 3e will develop into ridges with the increase in the compression strain. Figs. $3 b-3 f$ show that the position of folds or ridges can be well controlled in the present system by tuning the spacing of fibers. However, in the conventional film/substrate system, it is difficult to control the positions of ridges (Fig. 1d) because they are sensitive to the imperfections in the system [10]. Figs. $3 c$ and $3 f$ demonstrate the intriguing period-tripling mode obtained by controlling the spacing of fibers. It is 
pointed out that when compressing a stiff-film/homogeneous-substrate, period-tripling mode is not energetic favorable in comparison with the sinusoidal mode at a relatively small compression strain, e.g., $\varepsilon=0.1$. However, such advanced modes as the period-tripling, period-quadrupling and even period-quintupling can all be achieved in the present system at small or moderate compression strains by modulating the spacing of fibers.

$$
\mu_{p} / \mu_{m}=500 \quad \mu_{p} / \mu_{m}=0.01
$$

(a)

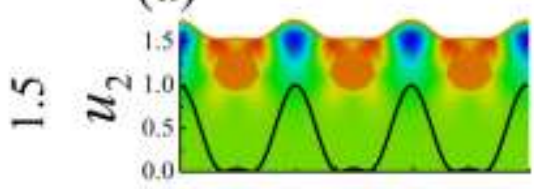

(b)

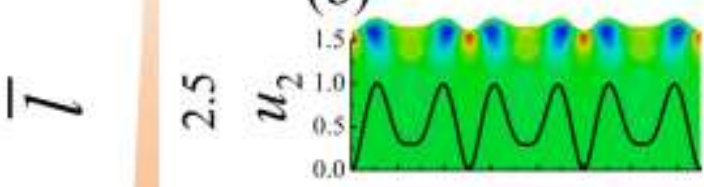

(c)

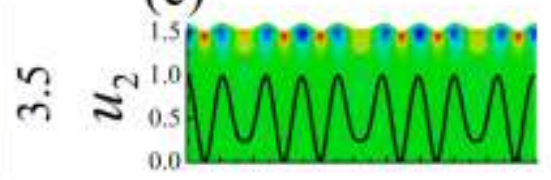

(d)

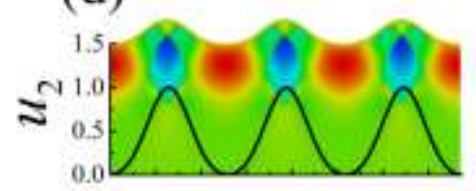

(e)

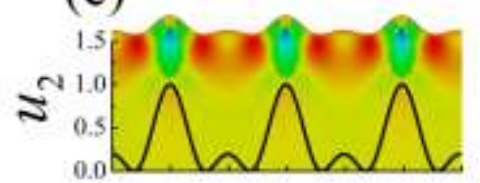

(f)

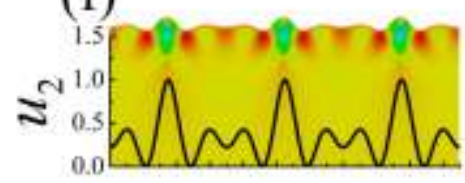

Fig. 3 Wrinkling patterns for different $\bar{l}$ for both soft fibers and stiff fibers, where $\mu_{\mathrm{f}} / \mu_{\mathrm{m}}=500, d / t=5, \lambda_{\mathrm{c}} / t=34.5, H / \lambda_{\mathrm{c}}=10, R / \lambda_{\mathrm{c}}=0.25$, the overall compression strain in all cases is $\varepsilon=0.1$. The solid lines in this figure and the following Figs. 4 and 5 illustrate the surface profiles of the deformed bilayers.

We proceed to study the effects of the cross-section dimension of fibers on the surface wrinkling patterns. Taking the case in Fig. $2 b$ as an example, we change the 
cross-section dimension of fibers to investigate the variation in the wrinkling patterns. Fig. 4 shows the simulation results for $R$ varying from $0.05 \lambda_{\mathrm{c}}$ to $0.75 \lambda_{\mathrm{c}}$. When the $R$ is small, e.g., $R=0.05 \lambda_{\text {c }}$, the fibers has no pronounced influence on the wrinkling patterns. When $R$ varies from $0.15 \lambda_{\mathrm{c}}$ to $0.5 \lambda_{\mathrm{c}}$, the period-doubling mode due to the effects of fibers is relatively stable. However, if $R$ is sufficient large, say $R=0.75 \lambda_{\text {c }}$, period-doubling mode disappears and ridge may occur between two neighboring fibers.
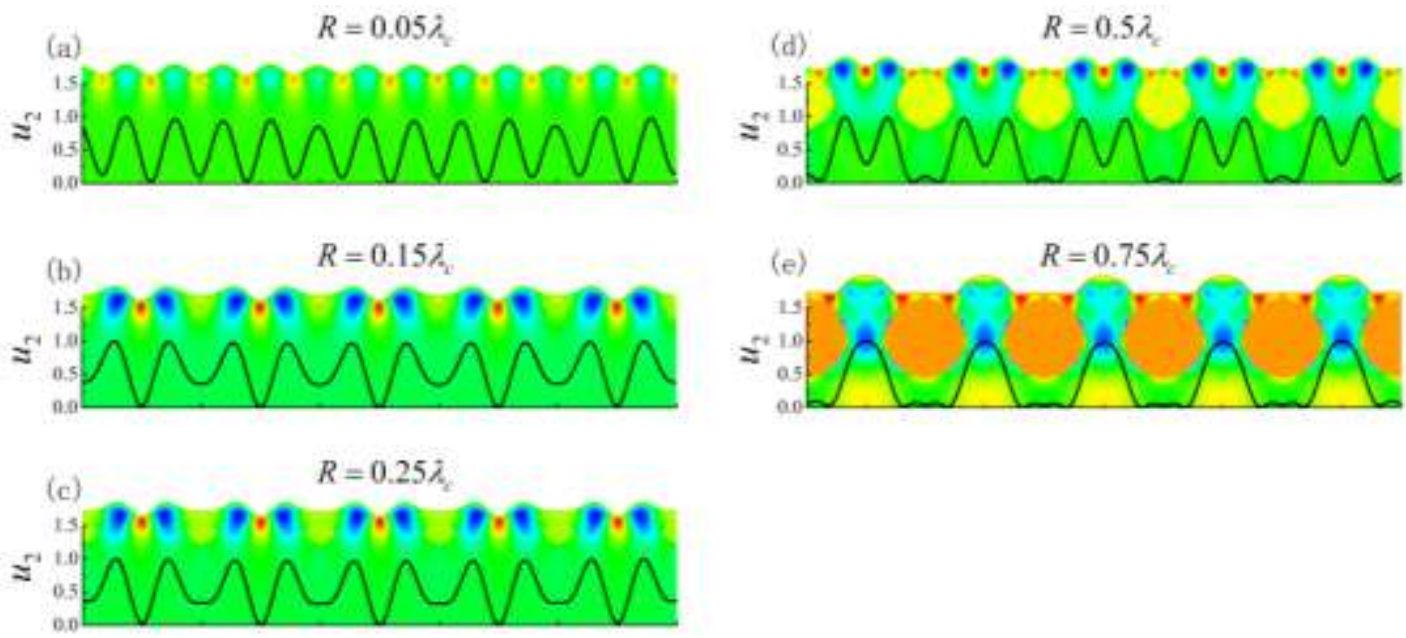

Fig. 4 Wrinkling patterns of the bilayer system with the fiber radius varying from $0.05 \lambda_{\mathrm{c}}$ to $0.75 \lambda_{\mathrm{c}}$.

The position of fibers, e.g., their distance from the interface, also has marked effects on the surface wrinkling patterns. Still taking the case in Fig. $2 b$ as an example, we change the position of fibers from $d=0.1 \lambda_{\mathrm{c}}$ to $d=2 \lambda_{\mathrm{c}}$, to investigate the variation in the wrinkling patterns. Fig. 5 shows the finite element results. It can be seen that when the fibers are near to the interface, e.g., $d=0.1 \lambda_{\mathrm{c}}$ and $d=0.2 \lambda_{\mathrm{c}}$, the period-doubling mode is rather stable (Figs. 5a and 5b). However, when the distance 
increases to $d=0.5 \lambda_{\mathrm{c}}$, the period-doubling and period-tripling modes co-exist and emerge alternatively (Fig. 5c). When the fibers are far from the interface, e.g., $d=2 \lambda_{\mathrm{c}}$, the effects of fibers on the surface wrinkling patterns are basically negligible (Fig. 5e).
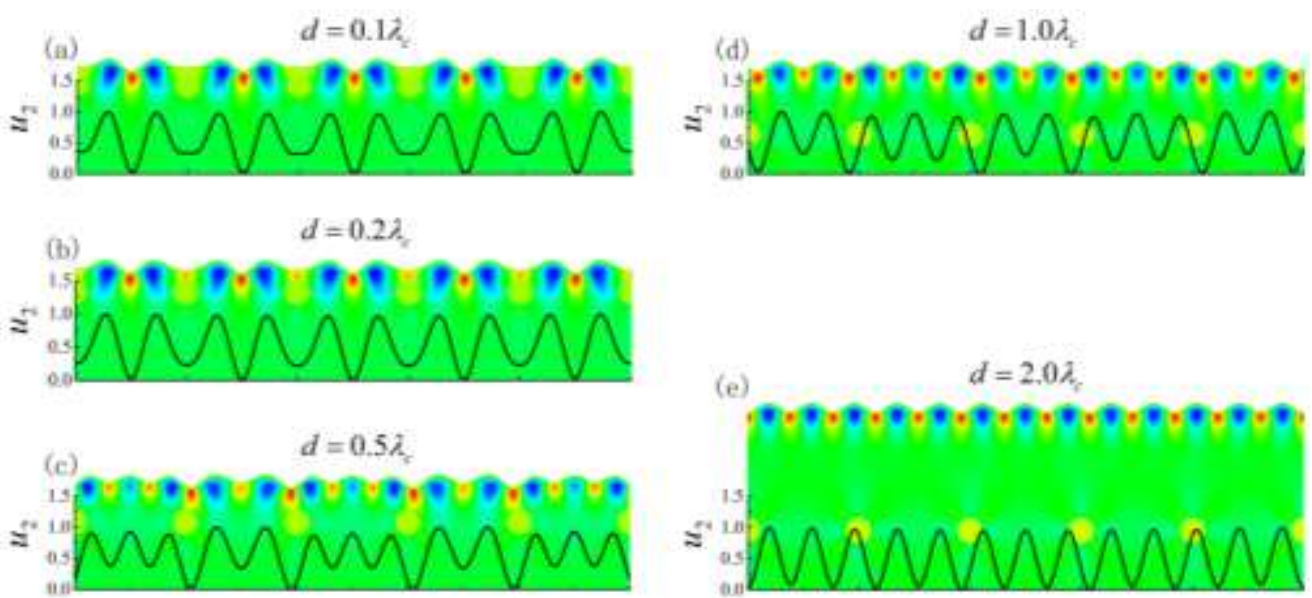

Fig. 5 Wrinkling patterns of the bilayer with the distance of fibers from the interface varying from $d=0.1 \lambda_{\mathrm{c}}$ to $d=2 \lambda_{\mathrm{c}}$.

\section{Band structures of the wrinkled bilayer system}

When the bilayer system is subjected to compression, surface wrinkling occurs and thereby periodic stress patterns can be generated depending on the magnitude of deformation and the fiber dimension, spacing and positions. The periodic stress patterns might have marked effects on the elastic wave band structures of the system and are investigated here.

For illustration, we take $H / \lambda_{\mathrm{c}}=1, \quad \mu_{\mathrm{f}} / \mu_{\mathrm{m}}=500, \quad \mu_{\mathrm{m}}=1 \mathrm{MPa}$, and $\rho_{\mathrm{f}}=\rho_{\mathrm{p}}=\rho_{\mathrm{m}}=1000 \mathrm{~kg} / \mathrm{m}^{3}$. Analysis is performed on the deformed configuration shown 
in Fig. 3, in which the compression strain is taken as 0.10 . According to the periodicity of the wrinkled structure, we take the representative volume elements (RVE) in Fig. 6 for different wrinkling patterns to conduct the analysis. The band structures of the bilayer systems are calculated by imposing the Bloch-type boundary conditions.

According to the Bloch's theorem [33], the small amplitude elastic waves in periodic structures can be expressed as

$$
\mathbf{u}(\mathbf{X}, t)=\phi(\mathbf{X}) e^{i(\mathbf{K} \cdot \mathbf{X}-\omega t)},
$$

where $\phi(\mathbf{X})$ is a periodic function with the same periodicity as the periodic structures, i.e., $\phi\left(\mathbf{X}+l \mathbf{E}_{x}\right)=\phi(\mathbf{X})$ (see Fig. 1), where $\mathbf{E}_{x}$ is the base vector along the $x$ direction in the undeformed configuration and $l \mathbf{E}_{x}$ is the lattice vector [33]. $\omega$ and $\mathbf{K}$ are the angular frequency and the wave vector, respectively. Accordingly, we obtain

$$
\mathbf{u}^{\mathrm{r}}=\mathbf{u}^{1} e^{i \mathbf{K} \cdot \mathbf{I} \mathbf{E}_{x}},
$$

where $\mathbf{u}^{\mathrm{r}}$ and $\mathbf{u}^{1}$ stand for the displacements of the right and left side of the RVEs, respectively. Eq. (5) is the so-called Bloch-type boundary condition. In the present study, $\mathbf{K}=k \mathbf{E}_{x}$ is parallel to the lattice vector, and $k$ varies continuously from $-\pi / l$ to $\pi / l$ when $\mathbf{K}$ sweeps through the first Brillouin zone. Utilizing Eq. (3), the band structure is calculated with the finite element method in the present study (see Appendix A for details).

For comparison, the band structures of a conventional bilayer system are first calculated, and the results are shown in Fig. 6a. We further investigate the band structures of the buckled bilayers with inhomogeneous substrates. Bloch wave analysis is performed on the stressed unit cell in the deformed configuration and the results are 
shown in Figs. 6b-6g. An important and key finding revealed by the results in Fig. 6 is that the bandgaps largely depend on the physical and geometrical parameters of the system and the compression strain in the present system. Therefore, tuning the physical and geometrical parameters and/or controlling the imposed external mechanical load enable achieving desired bandgaps.
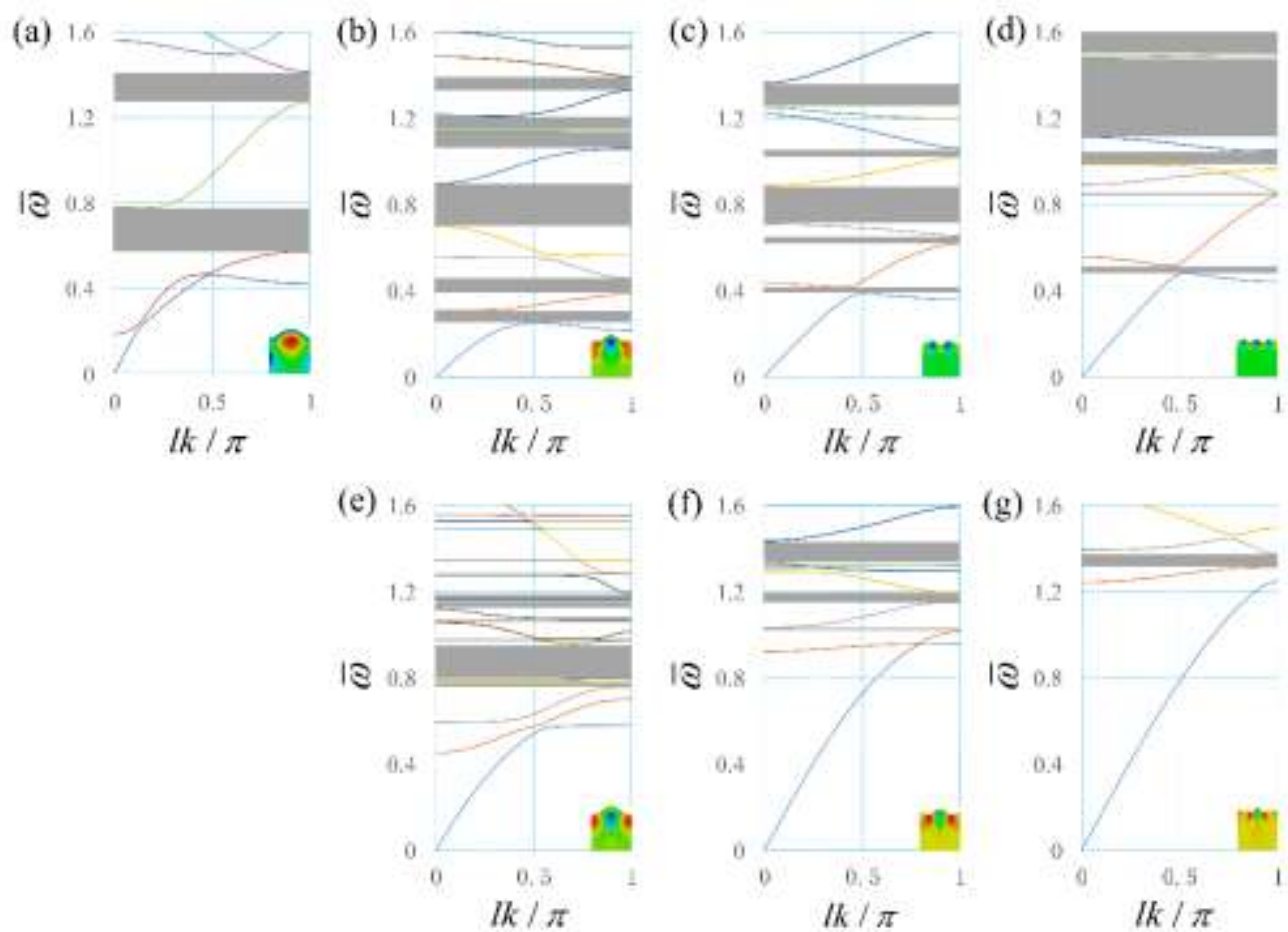

Fig. 6 The band structures for sinusoidal wrinkle pattern and the wrinkling patterns in Fig. 3. (a) shows the band structures for the conventional bilayer system. (b)-(g) represent the band structures of the deformed unit cells with stress patterns shown in Figs. 3a-3f. The overall compressive strain of $\varepsilon=0.1$ is imposed to the system in the analysis. The grey areas show the bandgaps. In the figure, $\bar{\omega}=2 \pi f l / \sqrt{\mu_{\mathrm{eff}} / \rho_{\mathrm{m}}}$, $\mu_{\text {eff }}=\left(\mu_{\mathrm{f}} A_{\mathrm{f}}+\mu_{\mathrm{m}} A_{\mathrm{m}}+\mu_{\mathrm{p}} A_{\mathrm{p}}\right) /\left(A_{\mathrm{f}}+A_{\mathrm{m}}+A_{\mathrm{p}}\right), A_{\mathrm{f}}, A_{\mathrm{m}}, A_{\mathrm{p}}$ are the cross-section areas of the film, substrate and fiber, respectively. $l$ is the length of the unit cell, $f$ is the circular frequency given by the natural frequency analysis with ABAQUS [31]. 
Distinct from the bilayer with a homogeneous substrate, band gaps exist in the composite system concerned here when no external load is applied (see Fig. 8a). Those band gaps result from the periodically distributed fibers in the matrix. When the composite is compressed and instability triggered, some new band gaps emerges, e.g., the 2nd and 4th band gaps as shown in Fig. 8b. These new emerged band gaps are induced by the periodic stress pattern in the deformed structure similar to those obtained in the wrinkled bilayer with a homogeneous substrate (see Fig. 7). As revealed in the previous studies [23,29], the first band gap in the bilayer with a homogeneous substrate mainly relies on the stress pattern instead of the magnitude of the stress. Accordingly, this band gap is stable when the external load increases (see Fig. 7 when $\varepsilon$ varies from 0.02 to 0.1 ) and no wrinkling pattern transition occurs. The 4 th band gap in Fig. 8b shares the similar feature, whereas other band gaps such as the 2nd band gap appear to be sensitive to the magnitude of the external load, and a new band gap emerges between the 1 st and 2 nd band gaps when $\varepsilon$ varies from 0.02 to 0.1 , indicating additional band gaps may emerge in the present system with the variation in the external compressive load. To conclude, some of the band gaps in the present system are insensitive to the magnitude of the external load and are relatively stable during the loading procedure, whereas some others vary with the external load and can be actively tuned by modulating the external stimuli in a practical design. 

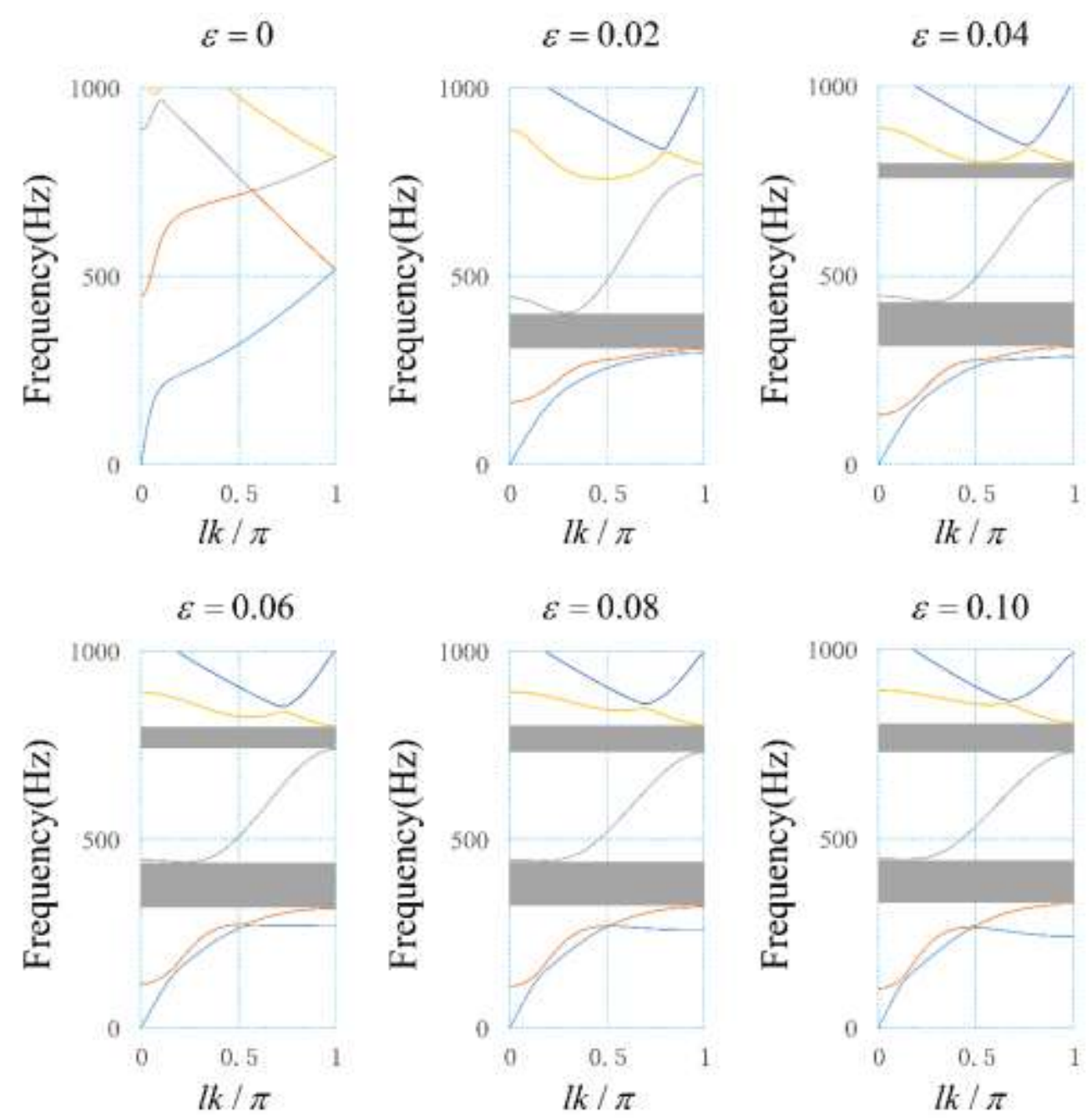

Fig. 7 Effects of the magnitude of deformation on the band structures in conventional bilayers with the overall compression strain $\varepsilon$. For $\varepsilon=0$, no bandgaps exist. 

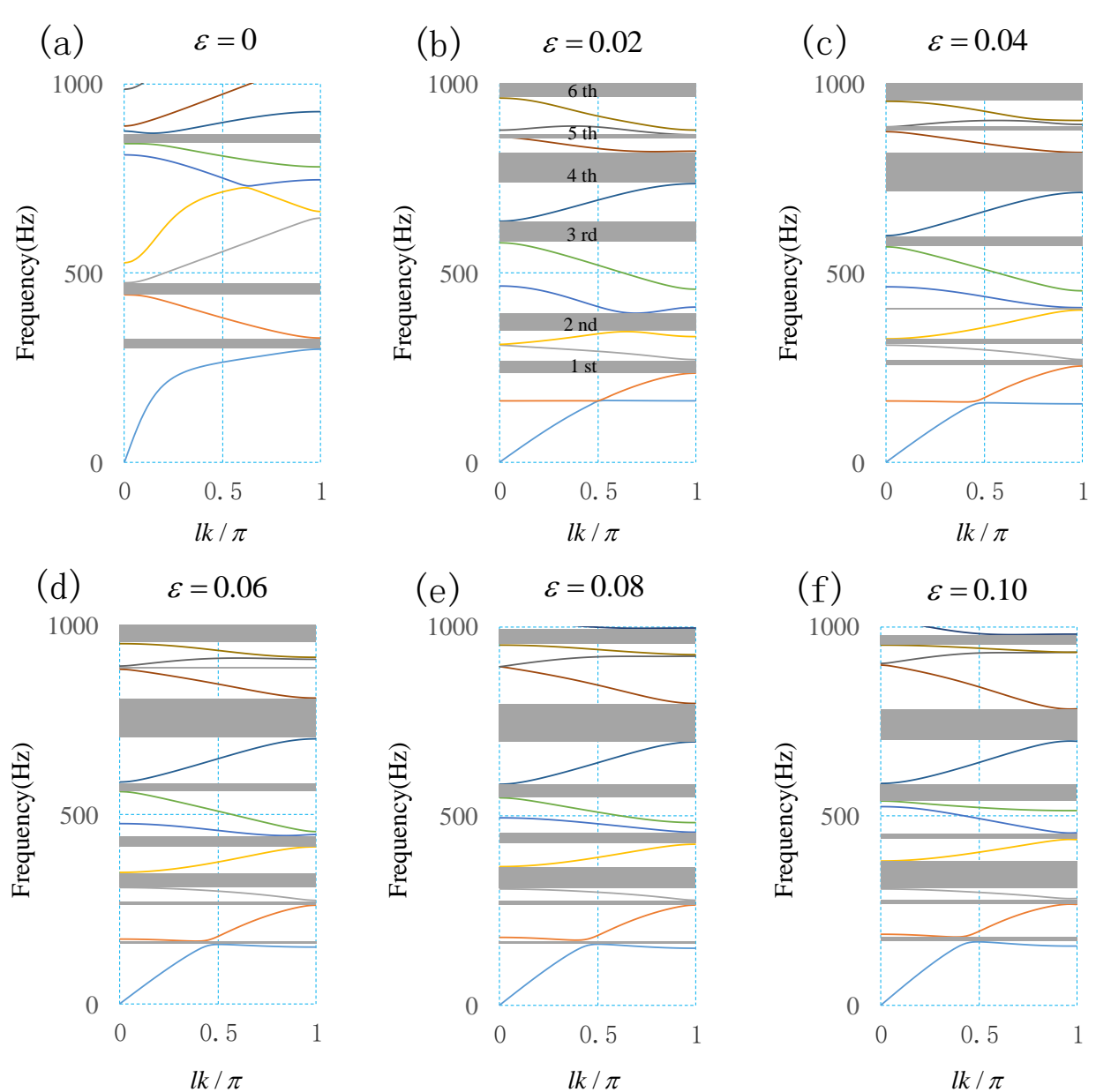

Fig. 8 Effects of deformation on the bandgap structures in the present stiff-film/fiber-filled substrate bilayer system. The bandgaps when $\varepsilon=0$ are due to the existence of fibers. Here the pattern in Fig. $6 \mathrm{c}$ is taken as an example. In (f) the frequency range at $\varepsilon=0.1$ is different from that in Fig. $6 c$, therefore more bandgaps are observed here.

\section{Transmission spectra}

To confirm the results in Fig. 6 given by the Bloch wave analysis, the transmission spectra of the composite structures are investigated with finite element analysis. The transmission coefficient is defined as [26,34] 


$$
c_{T}=20 \lg \left(\left|u_{1}^{\text {out }}\left(f_{\mathrm{h}}\right) / u_{1}^{\text {in }}\left(f_{\mathrm{h}}\right)\right|\right),
$$

where $u_{1}^{\text {in }}\left(f_{\mathrm{h}}\right)$ is the harmonic prescribed displacement at one side of the finite element model, and $u_{1}^{\text {out }}\left(f_{\mathrm{h}}\right)$ is the steady-state dynamic response received at the other side of the model. $f_{\mathrm{h}}$ is the frequency of the prescribed displacement. The FE analysis is conducted using the steady-state dynamics analysis step in Abaqus [31].

Taking the wrinkling mode shown in Fig. $6 \mathrm{c}$ as an example, we calculate the transmission spectra of the wrinkled structure, which consists of 20 unit cells. The results in Fig. 9 clearly shows that the frequency ranges corresponding to the drop of transmission are in good agreement with the bandgaps given by the Bloch wave analysis, indicating that our analysis based on the Bloch wave theory is reliable.

(a)

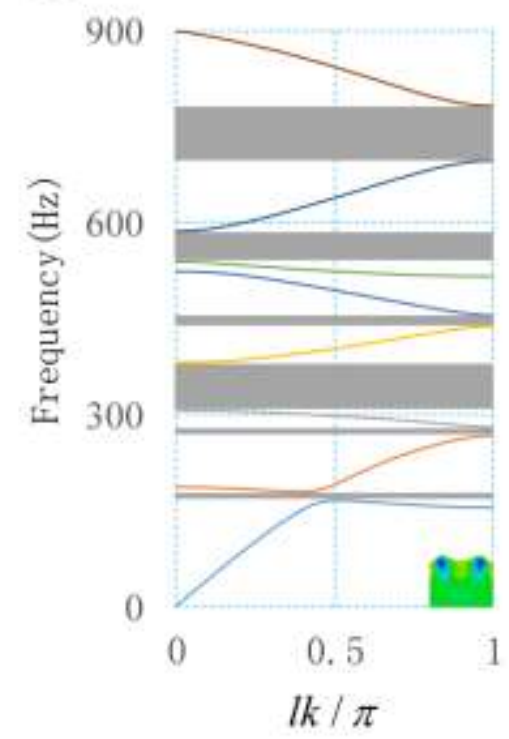

(b)

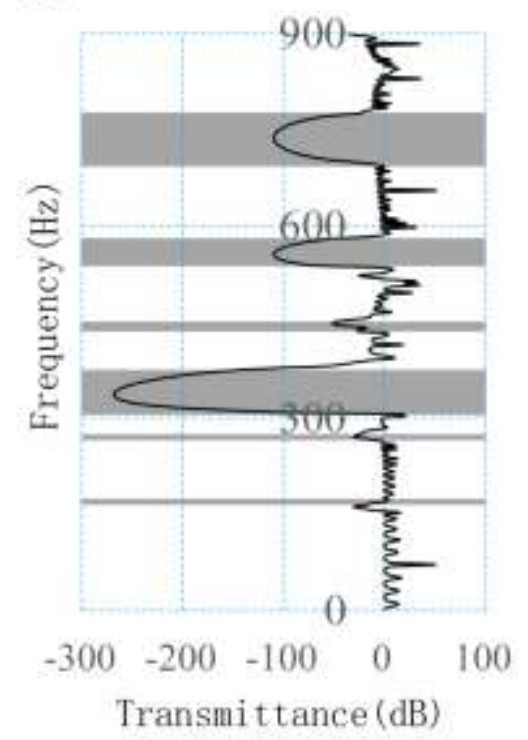

Fig. 9 Calculation of the transmission spectra. The transmission coefficient is defined as $20 \lg \left(\left|u_{1}^{\text {out }}\left(f_{\mathrm{h}}\right) / u_{1}^{\text {in }}\left(f_{\mathrm{h}}\right)\right|\right)$. The bandgaps shown in (a) are in good agreement with the strongly attenuated regions given by the lines in (b). 


\section{Conclusion}

In this paper, we study the wrinkling phenomena of a stiff film resting on a fiber-filled soft substrate and demonstrate its potential applications in the design of tunable soft metamaterials. In summary, the following key results have been achieved.

First, a nonlinear finite element model has been built to investigate the effects of the cross-section dimension and spacing, and positions of fibers embedded in the soft substrate on the wrinkling patterns of the system. We show that diverse wrinkling patterns including sinusoidal stripes, period-doubling and period-tripling wrinkles, folds and mountain ridges may occur due to the nonlinear and inhomogeneous deformation in the substrate. More importantly, they can be well controlled by tuning the cross-section dimension, spacing and positions of fibers.

Second, to illustrate the potential use of the diverse wrinkling patterns revealed in the present system, we investigate the elastic wave propagation in the wrinkled bilayer using the Bloch wave theory. Our computational results show that diverse stress patterns generated in the wrinkled bilayers give rise to a variety of band structures. In particular, our analysis reveals that desired bandgaps of the elastic waves can be achieved by designing the geometrical and physical parameters and/or controlling the external stimuli.

Third, to confirm the results given by the Bloch wave analysis, the transmission spectra of the buckled composite structures are investigated with finite element analysis. The results show that the frequency ranges corresponding to the drop of transmission 
are in good agreement with the band gaps given by the Bloch wave analysis.

The analysis and results reported here clearly indicate that the composite bilayer systems can serve as soft active metamaterials and may find broad technological applications for instance in the control of elastic waves.

\section{Acknowledgments}

The authors acknowledge financial support from the National Science Foundation of China (Nos. 11172155, 11572179, and 11432008). 


\section{Appendix A: Finite element method to calculate the band structures}

Following the technique develop by Åberg and Gudmundson [35], the dispersion relations of the periodic structures can be solved by commercial finite element codes with Bloch-type boundary conditions. The complex expression of $\mathbf{u}$ in Eq. (3) can be written as

$$
\mathbf{u}=\mathbf{u}_{\mathrm{Re}}+i \mathbf{u}_{\mathrm{Im}},
$$

where the subscripts 'Re' and 'Im' are the real and imagery part of $\mathbf{u}$, respectively. Inserting Eq. (A1) into Eq. (3) gives

$$
\begin{aligned}
& \mathbf{u}_{\mathrm{Re}}^{\mathrm{r}}=\mathbf{u}_{\mathrm{Re}}^{1} \cos (k l)-\mathbf{u}_{\mathrm{Im}}^{1} \sin (k l) \\
& \mathbf{u}_{\mathrm{Im}}^{\mathrm{r}}=\mathbf{u}_{\mathrm{Re}}^{1} \sin (k l)+\mathbf{u}_{\mathrm{Im}}^{1} \cos (k l)
\end{aligned}
$$

To implement Eq. (A2) in the Abaqus, two models with the same mesh are used. One stands for the real part of $\mathbf{u}$, and the other describes the imaginary part [35,36]. Eq. (A2) is then used to relate the two models, that is the nodes on the boundaries of the two models are linked by multi-point constraint in Abaqus [31]. For each wave vector in the first Brillouin zone, natural frequency analysis is conducted to obtain the corresponding angular frequency $\omega$ and further the total band structures. 


\section{References}

[1] B. Li, Y.-P. Cao, X.-Q. Feng, H. Gao, Mechanics of morphological instabilities and surface wrinkling in soft materials: a review, Soft Matter 8 (2012), 5728-5745.

[2] E. Cerda, L. Mahadevan, Geometry and Physics of Wrinkling, Phys Rev Lett 90 (2003), 074302.

[3] A. Goriely, M. Ben Amar, Differential Growth and Instability in Elastic Shells, Phys Rev Lett 94 (2005), 198103.

[4] B. Li, Y.-P. Cao, X.-Q. Feng, H. Gao, Surface wrinkling of mucosa induced by volumetric growth: Theory, simulation and experiment, J Mech Phys Solids 59 (2011), 758-774.

[5] E. Sharon, M. Marder, H. Swinney, Leaves, Flowers and Garbage Bags: Making Waves Rippled fractal patterns on thin plastic sheets and biological membranes offer elegant examples of the spontaneous breaking of symmetry, American Scientist 92 (2004), 254-261.

[6] F. Brau, H. Vandeparre, A. Sabbah, C. Poulard, A. Boudaoud, P. Damman, Multiple-length-scale elastic instability mimics parametric resonance of nonlinear oscillators, Nat Phys 7 (2011), 56-60.

[7] J. Liu, K. Bertoldi, Bloch wave approach for the analysis of sequential bifurcations in bilayer structures, P Roy Soc A-Math Phy 471 (2015).

[8] J.-Y. Sun, S. Xia, M.-W. Moon, K. H. Oh, K.-S. Kim, Folding wrinkles of a thin stiff layer on a soft substrate, Proceedings of the Royal Society A: Mathematical, Physical and Engineering Science 468 (2012), 932-953.

[9] R. Zhao, T. Zhang, M. Diab, H. Gao, K. S. Kim, The primary bilayer ruga-phase diagram I: Localizations in ruga evolution, Extreme Mechanics Letters 4 (2015), 76-82.

[10] Y. Cao, J. W. Hutchinson, Wrinkling Phenomena in Neo-Hookean Film/Substrate Bilayers, J Appl Mech-T ASME 79 (2012), 031019-031019.

[11] J. Zang, X. Zhao, Y. Cao, J. W. Hutchinson, Localized ridge wrinkling of stiff films on compliant substrates, J Mech Phys Solids 60 (2012), 1265-1279.

[12] Q. Wang, X. Zhao, Beyond wrinkles: Multimodal surface instabilities for multifunctional patterning, MRS Bulletin 41 (2016), 115-122.

[13] Y.-P. Cao, F. Jia, Y. Zhao, X.-Q. Feng, S.-W. Yu, Buckling and post-buckling of a stiff film resting on an elastic graded substrate, Int J Solids Struc. 49 (2012), 1656-1664.

[14] J.-W. Wang, B. Li, Y.-P. Cao, X.-Q. Feng, Surface Wrinkling Patterns of Film-Substrate Systems With a Structured Interface, J Appl Mech-T ASME 82 (2015), 051009-051009.

[15] F. Jia, Y.-P. Cao, T.-S. Liu, Y. Jiang, X.-Q. Feng, S.-W. Yu, Wrinkling of a bilayer resting on a soft substrate under in-plane compression, Philosophical Magazine 92 (2012), 1554-1568. 
[16] E. Lejeune, A. Javili, C. Linder, An algorithmic approach to multi-layer wrinkling, Extreme Mechanics Letters 7 (2016), 10-17.

[17] E. Lejeune, A. Javili, C. Linder, Understanding geometric instabilities in thin films via a multi-layer model, Soft Matter 12 (2016), 806-816.

[18] M. Guttag, M. C. Boyce, Locally and Dynamically Controllable Surface Topography Through the Use of Particle-Enhanced Soft Composites, Adv Funct Mater 25 (2015), 3641-3647.

[19] P. Wang, F. Casadei, S. Shan, J. C. Weaver, K. Bertoldi, Harnessing Buckling to Design Tunable Locally Resonant Acoustic Metamaterials, Phys Rev Lett 113 (2014), 014301.

[20] S. Shan, S. H. Kang, P. Wang, C. Qu, S. Shian, E. R. Chen, K. Bertoldi, Harnessing Multiple Folding Mechanisms in Soft Periodic Structures for Tunable Control of Elastic Waves, Adv Funct Mater 24 (2014), 4935.

[21] B. Alireza, G. Faramarz, Switching band-gaps of a phononic crystal slab by surface instability, Smart Mater Struct 24 (2015), 075009.

[22] S. Rudykh, M. C. Boyce, Transforming Wave Propagation in Layered Media via Instability-Induced Interfacial Wrinkling, Phys Rev Lett 112 (2014), 034301.

[23] G.-Y. Li, Y. Zheng, Y. Cao, X.-Q. Feng, W. Zhang, Controlling elastic wave propagation in a soft bilayer system via wrinkling-induced stress patterns, Soft Matter 12 (2016), 4204-4213.

[24] T. Brunet, A. Merlin, B. Mascaro, K. Zimny, J. Leng, O. Poncelet, C. Aristégui, O. Mondain-Monval, Soft 3D acoustic metamaterial with negative index, Nat Mater 14 (2015), 384-388.

[25] G. Ma, P. Sheng, Acoustic metamaterials: From local resonances to broad horizons, Science Advances 2 (2016).

[26] S. Babaee, N. Viard, P. Wang, N. X. Fang, K. Bertoldi, Harnessing Deformation to Switch On and Off the Propagation of Sound, Adv Mater (2015).

[27] Y. Huang, W. Q. Chen, Y. S. Wang, W. Yang, Multiple refraction switches realized by stretching elastomeric scatterers in sonic crystals, AIP Adv 5 (2015), 027138.

[28] Y. Tang, G. Lin, L. Han, S. Qiu, S. Yang, J. Yin, Design of Hierarchically Cut Hinges for Highly Stretchable and Reconfigurable Metamaterials with Enhanced Strength, Adv Mater 27 (2015), 7181-7190.

[29] G.-Y. Li, Y. Zheng, Y. Cao, Tunable defect mode in a soft wrinkled bilayer system, Extreme Mechanics Letters.

[30] X. Chen, J. W. Hutchinson, Herringbone Buckling Patterns of Compressed Thin Films on Compliant Substrates, J Appl Mech-T ASME 71 (2004), 597-603.

[31] ABAQUS, Analysis Theory manual, (2010).

[32] Y. Zhao, Y. Cao, W. Hong, M. K. Wadee, X.-Q. Feng, Towards a quantitative understanding of period-doubling wrinkling patterns occurring in film/substrate bilayer systems, P Roy Soc A-Math Phy 471 (2015). 
[33] C. Kittel, Introduction to Solid State Physics (Wiley, New York, 2004), 8 edn.

[34] Y. Pennec, J. O. Vasseur, B. Djafari-Rouhani, L. Dobrzyński, P. A. Deymier, Two-dimensional phononic crystals: Examples and applications, Surf Sci Rep 65 (2010), 229-291.

[35] M. Åberg, P. Gudmundson, The usage of standard finite element codes for computation of dispersion relations in materials with periodic microstructure, $\mathrm{J}$ Acoust Soc Am 102 (1997), 2007-2013.

[36] K. Bertoldi, M. C. Boyce, Wave propagation and instabilities in monolithic and periodically structured elastomeric materials undergoing large deformations, Phys Rev B 78 (2008), 184107. 